\title{
Road-to-Health Booklet assessment and completion challenges by nurses in rural primary healthcare facilities in South Africa
}

\author{
T Win, ${ }^{1}$ MB BS, DMA, IDHA, MSc Med, MPH; M G Mlambo, ${ }^{2,3} \mathrm{PhD}$ \\ ${ }^{1}$ Community Partners International, Bahan Township, Yangon, Myanmar \\ ${ }^{2}$ Department of Institutional Research and Business Intelligence, Risk and Advisory Services, University of South Africa, Pretoria, South Africa \\ ${ }^{3}$ Department of Family Medicine and Primary Care, Faculty of Health Sciences, University of the Witwatersrand, Johannesburg, South Africa
}

Corresponding author: M G Mlambo (motlatso.mlambo@wits.ac.za; mlambmg@unisa.ac.za)

\begin{abstract}
Background. Appropriate completion of the Road-to-Health Booklet (RTHB) remains crucial as it facilitates the provision of essential packaged health services for the growth and well-being of children under 5 years old.

Objective. To assess the new RTHB completion and its utilisation challenges by nurses in rural primary healthcare (PHC) in the West Rand District, Gauteng Province, South Africa.

Methods. A concurrent mixed-method design was used to conduct a retrospective record review of the 75 RTHBs completed by the nurses in the two PHC facilities. Nine in-depth interviews were also conducted with purposefully selected nurses to explore their RTHB utilisation challenges. Categorical data were analysed descriptively, comparing 'fully completed', 'partially completed' and 'not completed' sections of the RTHB. In-depth interviews were analysed using a thematic analysis approach which involved conducting inductive coding, grouping codes according to their similarity and identifying emerging themes.

Results. The highest completed sections of the RTHB were the weight-for-age growth chart (81\%) and mother PMTCT/HIV (78\%). The lowest completed section was the oral health examination, with only one booklet completed (7\%). Length/height $\times$ age $(37 \%)$ and weight $\times$ length/height $(33 \%)$ charts were partially completed. RTHB utilisation challenges included healthcare provider dilemmas (lack of time, burnout, habitual practices); patient-related dilemmas (language barriers, tampering with RTHB pages, lack of resources); and health system dilemmas (equipment shortages and lack of training for health personnel).

Conclusion. Although the quality of the RTHB is improving, there are still considerable challenges concerning its completion. It is therefore necessary to implement ways to improve its utilisation in this rural district, for effective under- 5 child health management. Without substantial improvement, opportunities for enhancing under- 5 child health will be missed.
\end{abstract}

S Afr J Child Health 2020;14(3):124-128. https://doi.org/10.7196.SAJCH.2020.v14i3.1685

The premature death of children under 5 remains a major challenge worldwide owing to poor child growth monitoring. ${ }^{[1]}$ Malnutrition is one of the significant contributors to the death of under-5 children, ${ }^{[2]}$ while also leading to poor child growth. In developing countries, an estimated 6.3 million under-5 children die as a result of common diseases which are highly treatable such as diarrhoea, malnutrition, pneumonia etc. ${ }^{[3]}$ Globally, growth monitoring and promotion (GMP) has been noted as a crucial intervention for improving the nutritional status, health services utilisation and mortality reduction of under-5 children. ${ }^{[4-6]}$ The African continent remains worst affected by the highest mortality rate of under- 5 children, accounting for above 100 per 1000 live births, ${ }^{[7]}$ with South Africa's (SA) rate estimated at $37-40$ per 1000 live births. ${ }^{[8]}$ Sustainable Development Goal (SDG) number 3.2 emphasises the reduction of neonatal mortality and under-5 mortality to at least 12 and 25 , respectively, per 1000 live births in all countries. ${ }^{[9]}$ Although child mortality rates have come down in SA, satisfactory data are still needed to assist with further reductions. ${ }^{\left[{ }^{[]}\right.}$

In SA, the Road-to-Health Card (RTHC) has been used as an essential monitoring tool for under-5 child health programmes since its introduction in 1973. In 2011, the Road-to-Health Booklet (RTHB) replaced the RTHC as an advanced comprehensive health system tool for monitoring child development and preventing under-5 mortality and morbidity in the context of primary healthcare. The main changes between the RTHB and the previous RTHC relate to three growth charts (weight-for-age, height-for-age and weight-for-height). These growth charts assist in monitoring child growth, focusing on stunted/severely stunted, wasted/severely wasted, and overweight/obese children. The charts also provide an opportunity for timeous checking of child development by using the developmental screening section of the RTHB. ${ }^{[10]}$ It is therefore crucial to ensure accurate RTHB data recording for the timely provision of interventions.

Although the Department of Health of SA has recognised the RTHB as ideal for the provision of crucial packaged health services for the growth and well-being of under-5 children, ${ }^{[1]}$ an array of challenges abound. A considerable number of problems relate to poor completion of the length/height $\times$ age and weight $\times$ length/ height growth charts; non-measurement of head circumference; ${ }^{[12,13]}$ non-completion of the mid-upper arm circumference (MUAC), developmental screening, deworming and oral health referrals sections; staff shortages and lack of equipment; ${ }^{[12-14]}$ missed immunisation opportunities; vitamin A non-provision; and no growth monitoring, feeding assessment and provision of nutritional advice. ${ }^{[13]}$ Other studies conducted in SA largely focused on specific sections of the RTHC/RTHB such as oral health, ${ }^{[14]}$ developmental screening and vitamin $\mathrm{A}^{[15]}$ promotion sections; ${ }^{[16]}$ and growth charts/MUAC sections. ${ }^{[17]}$ These studies also reflected significant 
challenges in completion of the RTHC and RTHB in various provinces. Lack of training could be the biggest contributor to poor completion of the RTHB. The present study assessed completion of the new RTHB and utilisation challenges experienced by nurses in the rural primary healthcare clinics (PHCs) of the West Rand District, Gauteng Province.

\section{Methods \\ Design and sampling}

This concurrent mixed-method study was conducted as part of a postgraduate degree ${ }^{[12]}$ to assess the new RTHB's completion and utilisation challenges experienced by nurses in the two rural PHC sites. The quantitative part of the study involved conducting a retrospective record review of the RTHB for all under- 5 children receiving child healthcare services in the two clinics between December 2014 and January 2015. The quantitative retrospective record review was used to provide information on under-5 children's growth parameters while also showing the level of RTHB completion (in terms of fully completed, partially completed and not completed sections). The sample size for the RTHB was calculated using the Department of Social Security's (DSS) research software formula, as shown below:

$$
\begin{aligned}
& n=\frac{Z^{2} 1-\infty / 2 p(1-p)}{d^{2}} \\
& d=\text { margin of error }=10 \% \\
& p=\text { coverage (completion rate }-50 \%)
\end{aligned}
$$

The population of under- 5 children in the two defined PHCs for the study period was 330 (279 and 51, respectively). Using a simple random sampling which involved taking samples every Monday and Friday, the overall sample size of 75 RTHBs (12 and 63) was considered appropriate.

In exploring RTHB utilisation challenges, the present study employed a purposeful sampling approach for selecting 10 nurses who participated in in-depth interviews. The tenth participant was, however, excluded owing to limited experience in managing under-5 children.

\section{Data collection}

A retrospective record review assessed completion of the 13 variables in the various sections of the booklet (excluding the immunisation section) using a data collection tick sheet and classifying them as fully completed, partially completed, not completed and not applicable. ${ }^{[12]}$ 'Fully completed' implied that all sections within the RTHB were entirely completed; 'partially completed' referred to non-completion of some sections within the booklet; 'not completed' referred to non-completion of sections within the booklet; and 'not applicable' referred to sections that were not meant to be completed because of the age of the child. Inclusion criteria were having all RTHBs of under- 5 children receiving child health services from the two selected clinics. ${ }^{[12]}$

The qualitative part involved conducting in-depth interviews with all the nurses (seven professional nurses, two enrolled nurses) providing child health services at the two designated PHCs to explore their RTHB utilisation challenges. Collecting such information enhanced the quantitative findings by providing reasons for lower completion rates in some sections of the RTHB. The in-depth interviews were conducted by two trained research assistants who were native speakers of the Setswana language spoken in the study area. Each interview was audio-recorded (after obtaining consent from the participants) and took place in a quiet and comfortable area provided by the facility manager, and it lasted for approximately 45 - 60 minutes. An interview guide consisting of questions and probes was used to guide interviews. In ensuring data quality, the study involved conducting two in-depth pilot interviews with nurses from other health facilities similar to the current study context.

\section{Data analysis}

Data from the retrospective record review were captured on Microsoft Excel 2010 (Microsoft Corp., USA), and exported to SPSS version 25 (StataCorp., USA) for descriptive analysis. Categorical data were compared according to different levels of RTHB completion: fully completed, partially completed, and not completed sections. A valid percentage was calculated by adding 'fully completed', 'partially completed' and 'not-completed' sections, but excluding 'not applicable'. A total percentage was calculated, including 'not applicable.'

In-depth interviews were transcribed verbatim. All interviews were conducted in English except for one that was later translated from Setswana to English. Data were analysed using a thematic analysis approach which involved conducting inductive coding, grouping codes according to their similarity, and identifying emerging themes. Rich participants' quotations were used to support the identified themes.

\section{Ethical considerations}

The University of the Witwatersrand's Human Research Ethics Committee (HREC) (M141040) and the West Rand District Research Committee gave ethical approval to conduct the study. The facility managers at the two sites also permitted the study. Upon receiving full information about the study, all participants consented to participate in the study. Retrospective record review took place after obtaining permission from the mothers of the under-5 children who were at the PHC at the time of the study. Confidentiality and anonymity for the record review data and the interview data were assured.

\section{Results}

\section{Status of the RTHB completion at the rural PHCs}

The quantitative section primarily focused on assessing the completion of 13 variables outlined in the RTHB (excluding the immunisation section that was fully completed). Table 1 reflects that the highest completed sections of the RTHB were weight-forage growth chart sections (81\%) and mother PMTCT/HIV (78\%), and the lowest completed section was the oral health examination with only one booklet completed (7\%). However, the length/height $\times$ age and weight $\times$ length/height charts were partially completed (37\% and $33 \%$, respectively). Head circumference was also partially completed (58\%). Forty-one percent of the child PMTCT/HIV was also not completed.

\section{Participants' demographic characteristics}

The second part of the study comprised only female participants $(100 \%)$ who were mostly professional nurses $(78 \%)$ with a few enrolled nurses (22\%). Most of them had 11 - 20 years' experience (56\%) in offering child health services, and a few had more than 20 years' experience $(44 \%)$. Only $56 \%$ of the participants attended the formal RTHB training.

\section{RTHB utilisation challenges in primary healthcare}

Three broad themes emerged depicting RTHB utilisation challenges, as outlined in Fig. 1. 
Table 1. RTHB completion status $(N=75)$

\begin{tabular}{|c|c|c|c|c|c|}
\hline $\begin{array}{l}\text { RTHB } \\
\text { section } \\
\text { number }\end{array}$ & Section & $\begin{array}{l}\text { Fully } \\
\text { completed, } n(\%)\end{array}$ & $\begin{array}{l}\text { Partially } \\
\text { completed, } n(\%)\end{array}$ & $\begin{array}{l}\text { Not } \\
\text { completed, } n(\%)\end{array}$ & $\begin{array}{l}\text { Not } \\
\text { applicable, } n(\%)\end{array}$ \\
\hline 1 & Well Child visits (pages $2-3$ ) & $58(77)$ & $16(22)$ & $1(1)$ & $0(0)$ \\
\hline 3 & Head circumference (page 5) & $41(58)$ & $2(3)$ & $27(39)$ & $5(7)$ \\
\hline 4 & Mother PMTCT/HIV (page 7) & $59(78)$ & $8(11)$ & $8(11)$ & $0(0)$ \\
\hline 5 & Child PMTCT/HIV (page 8) & $16(47)$ & $4(12)$ & $14(41)$ & $41(55)$ \\
\hline 6 & Vitamin A (page 9) & $32(65)$ & $3(6)$ & $14(29)$ & $26(35)$ \\
\hline 7 & Deworming (page 9) & $17(52)$ & $2(6)$ & $14(42)$ & $42(56)$ \\
\hline 8 & Developmental screening (page 13) & $42(62)$ & $8(12)$ & $18(26)$ & $7(9)$ \\
\hline 9 & Weight $\times$ age chart (pages $14-16$ ) & $61(81)$ & $5(7)$ & $9(12.0)$ & $0(0)$ \\
\hline 10 & Length/height $\times$ age (page 17) & $28(37)$ & $1(1)$ & $46(62)$ & $0(0)$ \\
\hline 11 & Weight $\times$ length/height (page 18 ) & $25(33)$ & $0(0)$ & $50(67)$ & $0(0)$ \\
\hline 12 & MUAC (page 19) & $38(57)$ & $4(6)$ & $25(37)$ & $8(11)$ \\
\hline 13 & Name of clinic visited (page 19) & $46(61)$ & $0(0)$ & $29(39)$ & $0(0)$ \\
\hline 14 & Oral health examination (page 20) & $1(7)$ & $0(0)$ & $13(93)$ & $61(81)$ \\
\hline
\end{tabular}

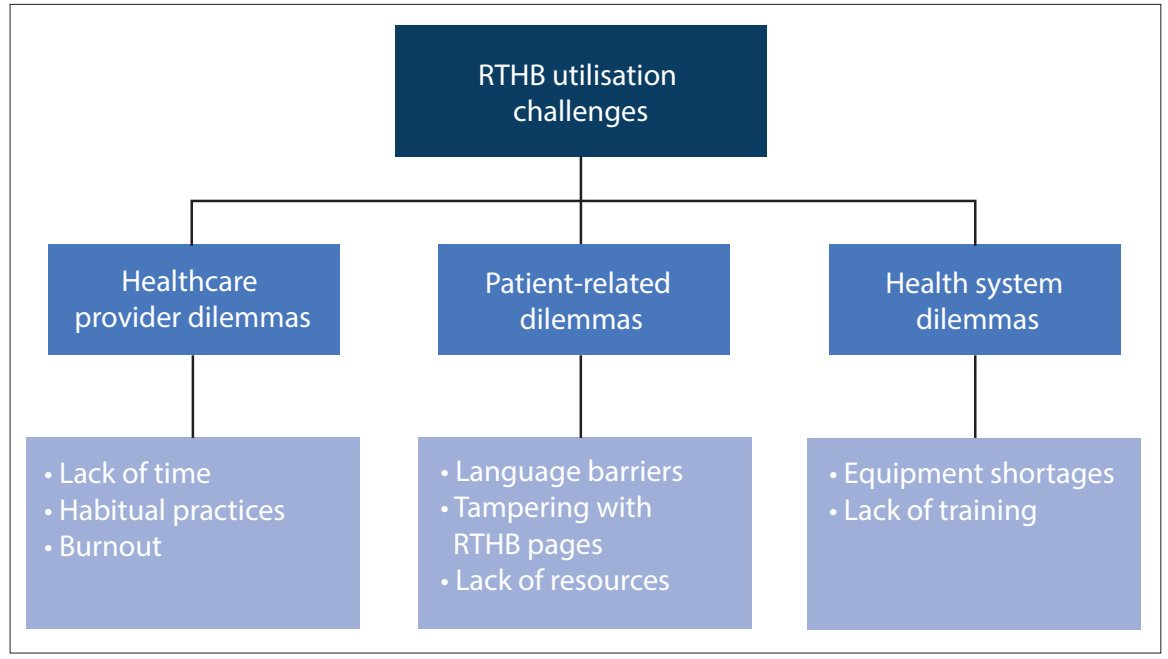

Fig. 1. RTHB utilisation challenges. (RTHB = Road-to-Health Booklet.)

Theme 1: Healthcare provider dilemmas

\section{- Lack of time}

Participants noted that RTHB completion dilemmas were due to lack of time on the healthcare provider's part. A participant said: '... there are a lot of things to be completed by the nurses. Some of the things I don't do as well.... you know what; we don't always have time, that is why this is not completed.' [P6, clinic 2; P1, clinic 2].

\section{- Habitual practices}

Most participants also highlighted challenges relating to the oral health and child HIV/PMTCT sections. Two participants reflected:

'... most of the books when you go to the oral health [section], this part is [usually] not filled in. [P8, clinic 1].

'... but like I said, I don't think you will get a lot of this books actually being completed.
I never see anybody fill this part [HIV/ PMTCT sections]' [P5, clinic 2].

\section{- Burnout}

Participants also mentioned the challenge of burnout amongst the health providers.

'So many things, first of all, our nurses are burned out... The burnout syndrome actually has got something to do with the shortage of staff... we are expected to give the same services and the same quality of care for the children and honestly, it is not practical...' [P4, clinic 2].

Another participant expressed challenges faced by big clinics:

'...But I'm just wondering about the big clinics where they are very busy with maybe one staff [member] because sometimes you'll find that it is [only] one staff [member] who is doing immunisation and then you have to write; you have to project; you have to check the documents...' [P6, clinic 2].

\section{Theme 2: Patient-related dilemmas}

- Language challenges

Most participants also noted challenges relating to the health promotion messages section that is written in English only. '...it is in English and most of our patients speak Tswana, and we have a lot of patients that speak Shangaan and Portuguese ...' [P1, clinic 1; P8, clinic 2].

\section{- Tampering with the RTHB pages}

Most participants also highlighted the child HIV/PMTCT information section as a challenge that mothers always raise.

'The HIV one. This is OK but parents/ mothers whoever, they sort of rub out this or tear off this page especially if they are HIV positive, they will tear off this page because they don't want people to see...' [P6, clinic 2].

\section{- Lack of resources}

Other patients raised challenges relating to a lack of resources.

'Then the oral health, most of the books when you go to oral health, this part is not filled in, because we refer them [to the hospital] and they don't go. They'll tell you that they don't have money and oral health is in town. [P7, clinic 2].

\section{Theme 3: Health system dilemmas \\ - Equipment shortage}

Participants further raised challenges of equipment non-availability that is useful in measuring length/height-for-age of children.

'With the length to height [length/heightfor-age], well, it's a prerequisite, it must 
be done. But given the circumstances where we are functioning, equipment is not there to do the length and height, especially with the ones that are above two years... but the others who are a little bit bigger... there's a challenge because our scale, the height thing is not up to standard.' [P3, clinic 2].

\section{- Lack of training}

'We didn't really understand being stunted, this thing, length for age, we didn't know them but now we know that if the child is on minus 2 , then they are stunted.' [Interview 8 , clinic 2].

\section{Discussion}

Our study reveals a higher completion rate of the weight-for-age growth chart (81\%). ${ }^{[12]}$ Similarly, other studies found more than $80 \%$ completion of the weight-for-age sections for both the RTHB and RTHC. ${ }^{[17,18]}$ However, other studies found lower completion of weight charts by nurses $(14.3 \%),{ }^{[16]}$ including inaccuracies in recording weight charts. ${ }^{[1,20]}$ The completion of this section is important as it facilitates early detection of underweight/severely underweight, stunted/severely stunted, wasted/severely wasted, and overweight/obese. ${ }^{[10,12]}$

Unlike other studies which found that height charts were incomplete in $91.2 \%$ of RTHBs, ${ }^{[16]}$ our study found only $37 \%$ completion of length/height-for-age and $33 \%$ completion of weightfor-length/height growth charts. In contrast, Jonker's study found high completeness of the length-for-age chart (79.6\%), compared with our study. ${ }^{[13]}$ Our qualitative findings reveal completion challenges relating to equipment shortages and lack of training for healthcare providers. Additionally, the challenges could be due to non-familiarity with new sections that were previously not included in the RTHC. Availability of resources, especially the equipment for measuring length or height, is vital to health service delivery. ${ }^{[21]}$ These challenges need to be addressed, as non-completion of such vital information suggests a missed opportunity by clinics to identify children who are stunted, wasted and overweight during their early stages of development.

Our study further reveals that neither clinic measured MUAC to a sufficient extent. Not knowing MUAC measurements suggests that nurses could not identify whether a child had severe or moderate malnutrition. Similarly, Koetaan et al's study in Free State Province found that only $11.7 \%$ of children were measured for MUAC. ${ }^{[17]}$ Training in this characteristic is necessary, as suggested by the qualitative findings in the current study. Our findings show that completion of head circumference was missed in more than onethird of the children. Similarly, head circumference was not recorded in $86 \%$ of the children in a study conducted in Makhado. ${ }^{[11]}$ In another study, only $3.6 \%$ of RTHBs documented head circumference of children at 14 weeks. ${ }^{[18]}$ The low level suggests a missed opportunity for early awareness of hydrocephaly and microcephaly.

The findings further reveal that the opportunity for early identification of developmental delays of children that could assist in preventing additional developmental challenges had been missed for one-third of the children. Furthermore, other studies found that no developmental assessments had been completed. ${ }^{[11,13]}$ The need for training in this regard is urgent.

Concerning the mother PMTCT/HIV information section, only $11 \%$ of the booklets were partially completed and others not completed at all (11\%), with some missing pages. Our qualitative findings highlight patient-related dilemmas, where mothers/caregivers hide their HIV-positive status. Additionally, $41 \%$ of children exposed to HIV did not have information recorded about PCR testing. Likewise, other studies reported significantly less HIV-related information had been recorded; ${ }^{[1]}$ this finding indicates an important missed opportunity for the improvement of PMTCT indicators.

In the present study, a total of 14 children required referrals for oral health service facilities; however, only one (1\%) was noted in the RTHB. Similarly, Cader and Naidoo's study found only three booklets were completed in the oral health section. ${ }^{[14]}$ This figure is explained by patient-related dilemmas, which note a lack of resources for patients to reach hospitals as the problem. Additionally, healthcare provider dilemmas owing to habitual practices affect non-completion of this section, which suggests a need for awareness creation on the importance of completing this section.

The present study further found $65 \%$ completion of the vitamin A section, which is higher than the national target of $60 \%{ }^{[22]}$ another study in Gauteng Province recorded (58.8\%) coverage. ${ }^{[13]}$ However, another study found that more than $80 \%$ of the children received vitamin A according to their ages. ${ }^{[12]}$ Poor administration of vitamin A could lead to detrimental effects on children, including death. ${ }^{[4]}$ The deworming section in the current study was only $51 \%$ completed, compared with the vitamin A section (65\%). These findings suggest that deworming is carried out much less than vitamin A supplementation, although the frequency of the schedule is more or less the same; this could be due to the mebendazole medication being out of stock. ${ }^{[12]}$

Our study also found partial completion of the Well Child Visits section (22\%). The Well Child Visits section is presented in the form of a checklist for the whole RTHB, suggesting that completion should have been fairly easy. Such partial completion suggests that nurses did not use this section appropriately as a checklist; therefore, training is of paramount importance in this regard. The need for further capacity building suggests that participants do not feel adequately competent to utilise the RTHB; hence the incomplete sections. Additionally, health promotion information is often missed owing to language challenges. It is therefore vital to make appropriate design and language changes that would still protect patients' privacy. The results suggest that the quality of the RTHB is improving compared with the previous RTHC; however, its completion is still significantly poor. Without substantial improvement, there will still be missed opportunities for under-5 children. It is essential to conduct RTHB/ growth parameters training for clinic nurses. Ongoing follow-ups are therefore necessary after training. Regular re-training/drills at individual clinics are also needed. Regular audits on completion of the RTHB should also be implemented. Further research is recommended on an holistic approach to cover the four sub-districts and assess the quality of plotting of the growth and MUAC charts. The District Clinical Specialist Team (DCST) should consider monitoring and evaluating a quality control programme in the district to develop guidelines for proper completion of the RTHB. The Plan-Do-Study-Action (PDSA) approach will be appropriate to assess problems that persist.

The present study has several limitations. It was conducted in only one district; therefore, findings cannot be generalised to other settings. Inaccuracies in the booklets might affect the quality of the results. The findings might also be biased as the two clinics were visited three months before commencement of the study by a DCST.

\section{Conclusion}

The study revealed utilisation challenges experienced by the two facilities in completing the new RTHB, except for the weight $\times$ age chart. It is therefore crucial to improve RTHB utilisation in the district as it is central to the management of under- 5 child health. 
Declaration. This manuscript was submitted in partial fulfilment of the requirements for a MPH degree.

Acknowledgements. We acknowledge Dr Richard Cooke, who was involved in supervision of the Master's study. Our sincere gratitude also goes to participants in the two clinics who allowed us to review the RTHBs and conduct interviews with them. We thank Papikie Makhuba and Mapula Adams, who were research assistants in the study.

Author contributions. TW conducted the study for a postgraduate degree supervised by MGM. TW conceptualised the study, collected data, analysed data and wrote the paper. MGM contributed to study conceptualisation, trained data collectors, analysed data, and participated in writing the paper.

Funding. None.

Conflicts of interest. None.

1. UNICEF. Learning from Nutrition Programme Evaluations: A Thematic Evaluation Synthesis Report. (2014). http://www.unicef.org/evaluation/files/ Final_learning_from_nutrition_17_07_2014.pdf (accessed 9 January 2015).

2. Kramer CV, Allen S. Malnutrition in developing countries. Paed Child Health 2015;25(9):422-427.

3. Vakili R, Emami Moghadam Z, Khademi G, Vakili S, Saeidi M. Child mortality at different world regions: A comparison review. Int J Pediatr 2015;3(4.2):809816. https://doi.org/10.22038/ijp.2015.4641

4. World Health Organization. Global health risks: Mortality and burden of disease attributable to selected major risks. Geneva: WHO; 2009.

5. Lutge E, Friedman I, Mbatha T. A review of health research in South Africa from 1994 to 2007: Primary health care: Systems support. S Afr Health Rev 2008; 1:211-221.

6. Goldfeld S, Yousafzai A. Monitoring tools for child development: An opportunity for action. Lancet Glob Health 2018;6(3):e232-e233. https://doi. org/10.1016/s2214-109x(18)30040-8

7. World Health Organization. Global Health Observatory (GHO) data: Child mortality and causes of death. https://www.who.int/gho/child_health/ mortality/mortality_under_five_text/en/ (accessed 10 January 2019).

8. Bamford LJ, McKerrow NH, Barron P, Aung Y. Child mortality in South Africa: Fewer deaths, but better data are needed. S Afr Med J 2018;108(3 Suppl 1):S25-S32. https://doi.org/10.7196/SAMJ.2018.v108i3.12779

9. World Health Organization. Sustainable Development Goals. https://www.who. int/sdg/targets/en/ (accessed 10 January 2019).
10. Martin P. The unrealised potential of the Road to Health. (August 2015) http:// ilifalabantwana.co.za/the-policy-post-the-unrealised-potential-of-the-road-tohealth-booklet/ (accessed 12 January 2019).

11. Kitenge G, Govender I. Nurses' monitoring of the Road to Health Chart at primary healthcare level in Makhado, Limpopo Province. S Afr Fam Pract 2013;55(3):275-280. https://doi.org/10.1080/20786204.2013.10874350

12. Win T. An assessment of the 'road-to-health-booklet' based on knowledge/ perceptions of the clinic nurses and conduct a record review of the completion of the booklets. 2016. http://hdl.handle.net/10539/22555

13. Jonker L, Stellenberg EL. Missed opportunities in child healthcare. Afr J Prim Health Care Fam Med 2014;6(1):1-8. https://doi.org/10.4102\%2Fphcfm.v6i1.537

14. Cader R, Naidoo S. The Oral Health Section of the Road to Health Chart (RtHC) - How useful is it? S Afr Dent J 2019;74(10):556-560.

15. Naborn B. The use of the road-to-health booklet developmental screening in the detection and referral of early developmental delay in the Pelonomi hospital drainage area [unpublished thesis]. Faculty of Health Sciences, University of the Witwatersrand; 2016.

16. Du Plessis LM, Koornhof HE, Marais ML, Blaauw R. Implementation of the Roadto-Health-Booklet health promotion messages at primary health care facilities, Western Cape Province, South Africa. S Afr J Child Health 2017;11(4):164-169. https://doi.org/10.7196/sajch.2017.v11i4.1414

17. Koetaan D, Smith A, Liebenberg A, et al. The prevalence of underweight in children aged 5 years and younger attending primary health care clinics in the Mangaung area, Free State. Afr J Prim Health Care Fam Med 2018;10(1):1-5. https://doi.org/10.4102/phcfm.v10i1.1476

18. Naidoo H, Avenant T, Goga A. Completeness of the Road-to-Health Booklet and Road-to-Health Card: Results of cross-sectional surveillance at a provincial tertiary hospital. S Afr J HIV Med 2018;19(1):1-10. https://doi.org/10.4102/ sajhivmed.v19i1.765

19. Tarwa C, De Villiers F. The use of the Road to Health Card in monitoring child health. S Afr Fam Pract 2007;49(1):15-15d. https://doi.org/10.1080/20786204.2 007.10873497

20. Cloete I, Daniels L, Jordaan J, Derbyshire C, Volmink L, Schubl C. Knowledge and perceptions of nursing staff on the new Road to Health Booklet growth charts in primary healthcare clinics in the Tygerberg sub-district of the Cape Town metropole district. S Afr J Clin Nutr 2013;26(3):141-146. https://doi.org/ $10.1080 / 16070658.2013 .11734458$

21. Mounier-Jack S, Griffiths UK, Closser S, Burchett H, Marchal B. Measuring the health systems impact of disease control programmes: A critical reflection on the WHO building blocks framework. BMC Public Health 2014;14(1):278. https:// doi.org/10.1186/1471-2458-14-278

22. Massyn N, Peer N, English R, Padarath A, Barron P, Day C, eds. District Health Barometer 2015/16. Durban: Health Systems Trust; 2016.

Accepted 9 January 2020 